\title{
Intellectual property rights and good research practice
}

\author{
Dennis A. Revicki · Carolyn E. Schwartz
}

Published online: 3 November 2009

(C) Springer Science+Business Media B.V. 2009

Who owns the rights to health outcome-related items and instruments? There are various responses to this question, and varying interpretations of what is meant by intellectual property rights. Intellectual property lawyers would likely have a complicated and involved interpretation and it is our understanding that collections of items can be copyrighted as instruments, but the question is less clear for the individual items. Does this mean that any researcher can take any collection of items from one or more existing instruments and create a new instrument? Yes, we think so, but (and this is an important but) the new instrument cannot be labeled as any of the existing instrument(s) used to derive the new form. Recently, a group of researchers examined the factor structure and psychometric qualities of the standardized version of the Asthma Quality of Life Questionnaire [1] and found that several of the items may not be relevant for the Greek translation of the instrument [2]. While the psychometric results are useful for understanding the measurement qualities of the AQLQ, the authors implied that they developed a new version of the instrument, the AQLQ-18. This resulted in an interesting exchange of perspectives related to intellectual property and copyright issues related to health outcome measures $[3,4]$.

D. A. Revicki $(\square)$

United Biosource Corporation, Bethesda, MD, USA

e-mail: Dennis.Revicki@unitedbiosource.com

C. E. Schwartz

Delta Quest Foundation Inc., Concord, MA, USA

e-mail: carolyn.schwartz@deltaquest.org

C. E. Schwartz

Tufts University Medical School, Boston, MA, USA
We thought that we would take this opportunity to express our views on copyright issues and good research practice related to the field of health-related quality of life research. The definition of copyright is the exclusive legal right to reproduce, publish, sell, or distribute the matter and form of something (as a literary, musical, or artistic work) (www.merriam-webster.com/dictionary accessed July 21, 2009). Basically, in the case of instrument developers, this represents the legal right to control the use and reproduction of the original instrument. The maintenance of the scientific integrity of the copyrighted instrument ensures researchers and readers of scientific journals that the study used the correct version of the instrument and that there is evidence supporting the psychometric qualities of the instrument. This also ensures a consistency in versions across multiple clinical trials and other studies.

Good research practice indicates that the relevant permission to use an instrument should be followed when deciding to use a HRQL or symptom-assessment instrument in a research study. Different instrument developers have different systems for registering use of their instrument for research purposes. Some of these systems require users to pay fees either by study or by individual subject, but not all instrument developers require payment. For example, the Patient-Reported Outcome Measurement Information System (PROMIS), a US National Institutes of Health sponsored initiative, makes all the developed and evaluated item banks and short-form measures publicly available and requires only completion of a standardized registration form [5; www.nihpromis.com]. Regardless, the instrument user should obtain permission from the developer before including a measure in a study. The research should also respect the scientific integrity of the instrument and not make modifications of the measure, but still retain the original copyrighted name of the instrument. A 
modified instrument can no longer be assumed to have the same evidence basis for reliability and validity as the original instrument [6]. Confusion in health outcomes research will reign if there are multiple versions of the same instrument being used in different research studies but which use the same instrument label. This has been a problem in translated health outcomes instruments for some time, where locally translated and adapted instruments are being used which differ from translated versions using more standardized methods [7].

HRQL research has for the past 50 years evolved through the investigation, adaptation and modification of earlier instruments to generate new and improved methods for measuring health outcomes. Preservation of copyrighted instruments helps maintain the pedigree and scientific integrity of health outcome measures and helps consumers of HRQL research fully understand this evolution. As HRQL research expands its focus, impact, and repertoire of tools, we all need to understand and accept the need to clearly document our instruments. We welcome informative commentary on these and other issues in Quality of Life Research and hope to encourage the publication of studies that exercise good research practices that advance health outcomes assessment for the future.

\section{References}

1. Juniper, E., Buist, S., Cox, F. M., Ferrie, P. J., \& King, D. R. (1999). Validation of a standardized version of the asthma quality of life questionnaire. Chest, 115, 1265-1270.

2. Grammatopolous, E., Skordilis, E., Koutsouki, D., \& Baltopoulos, G. (2009). An 18-item standardized asthma quality of life questionnaire-AQLQ(S). Quality of Life Research, 17, 323-332.

3. Juniper, E. (2009). Modification, translation and adaptation of questionnaires: Should copyright laws be observed? Quality of Life Research, 18(5), 531-533.

4. Grammatopolous, E., Skordilis, E., Koutsouki, D., \& Baltopoulos, G. (2009). An 18-item AQLQ(s) model: A response to Juniper. Quality of Life Research, 18(5), 535-539.

5. Cella, D., Yount, S., Rothrock, N., et al. (2007). The patientreported outcomes measurement information system (PROMIS): Progress of an NIH roadmap cooperative group through its first two years. Medical Care, 45(Suppl 1), S3-S11.

6. Coons, S. J., Gwaltney, C. J., Hays, R. D., et al. (2009). Recommendations on evidence needed to support measurement equivalence between electronic and paper-based patient-reported outcome (PRO) measures: ISPOR ePRO good research practices task force report. Value Health, 12, 419-429.

7. Wild, D., Grove, A., Martin, M., et al. (2005). Principles of good practice for the translation and cultural adaptation process for patient-reported outcomes (PRO) measures: Report of the ISPOR task force for translation and cultural adaptation. Value Health, 8, 94-104. 